\title{
Mechanical milling of the intermetallic compound AlFe
}

\author{
M. Meyer, L. Mendoza-Zélis, and F. H. Sánchez \\ Departamento de Física, Universidad Nacional de La Plata, C.C. 67, 1900 La Plata, Argentina \\ M. T. Clavaguera-Mora \\ Grup de Física dels Materials I, Departament de Física, Universitat Autonoma de Barcelona, 08193 Bellaterra, Spain \\ N. Clavaguera \\ Departament ECM, Facultat de Física, Universitat de Barcelona, Diagonal 647, 08028 Barcelona, Spain
}

(Received 4 January 1999)

\begin{abstract}
The intermetallic compound AlFe in its ordered $B 2$ phase has been ground under Ar in a ball-milling device. The system evolution under mechanical work has been examined as a function of time of milling by $\mathrm{x}$-ray diffraction, transmission Mössbauer spectroscopy, and differential scanning calorimetry. Short and long range disorder was observed. The evolution of the long range order parameter with milling time is analyzed up to the completion of the solid state reaction when a steady state value is reached. The short range order evolution is consistent with a nonstatistical order distribution throughout the sample but mainly distributed in two regions with different degrees of order each. Kinetic analysis of the reordering and recrystallization has been performed under continuous heating and isothermal calorimetric regimes. Modeling of these processes is presented as well as the estimated values of both the enthalpy and the activation energy of the reordering process. Results are compared with preceding information on related systems. [S0163-1829(99)07429-9]
\end{abstract}

\section{INTRODUCTION}

Disordering by mechanical work of bcc intermetallics with $\mathrm{B2}(c P 2-\mathrm{CsC})$ or $\mathrm{DO}_{3}\left(c F 16-\mathrm{BiF}_{3}\right)$ structure is a well known phenomenum and the stability of these compounds under mechanical milling has been extensively examined in the last decade. In the case of $\mathrm{AlFe}$, a pioneer work from Huffman et al. ${ }^{1}$ reported the modifications induced by severe cold work (crushing) in a series of $\mathrm{Al}_{1-x} \mathrm{Fe}_{x}(0.5 \leqslant x \leqslant 0.7)$ compounds. Their results indicated that crushing creates, through plastic deformation, a large number of antiphase boundaries (APB) leading to antiphase domains (APD) with average linear dimensions of about six atomic distances. As it is well known, plastic deformation takes place in this system mainly by the slip of $\{110\}$ planes in the $\langle 111\rangle$ direction.

Recently, Pochet et $a .^{2}$ presented a detailed study about the modifications induced by milling in $B 2-\mathrm{AlFe}$. It is rather well established that this compound, as well as B2-AlRu (Ref. 3) and B2-AlNi (Ref. 4), does not amorphize under milling but undergoes a transition to a metastable partially disordered state accompanied by a reduction in particle and domain sizes and accumulation of microstrain. Indeed, after prolonged milling, a steady state is reached which is characterized by a nonzero value of the long range order parameter that depends on temperature, composition, and milling intensity. ${ }^{2,5,6}$ This has been interpreted ${ }^{2}$ as an out of equilibrium order-disorder transition $B 2 \rightarrow A 2(c I 2-\mathrm{W})$ induced by the external mechanical forcing. Other authors ${ }^{7}$ have simply invoked the creation during the mechanical milling of excess antisite defects (AS), vacancies $(V)$, or combinations of them to explain the observed changes.

On the other hand, Monte Carlo simulations ${ }^{8}$ of the behavior of an ordered $B 2$ crystal under sustained shear has been attempted. When the effect of shearing is modeled by the forced slip of $\{110\}$ planes in the $\langle 111\rangle$ direction large fluctuations of the long range order parameter are obtained, linked with a bifurcation in microstructures which could be related to the observed order-disorder transition.

Mössbauer effect (ME) spectroscopy, may prove useful in the study of these mechanically induced transformations due to their ability of sensing the surroundings of a given isotope used as a probe. Indeed, from the ${ }^{57} \mathrm{FeME}$ spectra, information about the hyperfine fields: magnetic hyperfine field, electric quadrupole splitting $(\Delta)$, and the isomer shift $(\delta)$ at a given Fe site, can be obtained. The combination of this information with that coming from x-ray diffraction (XRD) should help to understand how the observed long range (LR) disorder correlates with short range (SR) structures. On the other hand, calorimetric studies allow to follow the time and temperature evolution of the several processes involved in the way back towards the equilibrium state giving indirect information about the nature of the induced disorder during milling.

We present here results on the mechanical milling of the intermetallic compound $B 2-\mathrm{AlFe}$ and on its further thermal annealing at moderate temperatures, obtained with ME spectroscopy, XRD, and differential scanning calorimetry (DSC). They were carried on with the aim of contributing to the understanding of the observed mechanically induced transformation to a metastable disordered state in $B 2$ intermetallics.

\section{EXPERIMENTAL}

The starting intermetallic compound AlFe was prepared by heating in evacuated $\left(p \leqslant 3 \times 10^{-6}\right.$ mbar) quartz ampoules, at $1000{ }^{\circ} \mathrm{C}$, a mixture of elemental pure Fe (Fluka 0.995 ) and Al (Johnson-Matthey 0.995 ) powders in equi- 
atomic proportion. The resulting ingot was ground and annealed in vacuum for $72 \mathrm{~h}$ at $550^{\circ} \mathrm{C}$ in order to remove the remaining stress and to improve the degree of order. Samples of $250 \mathrm{mg}$ each, together with one chrome steel ball $(\Phi$ $=9 \mathrm{~mm})$, were sealed into chrome steel cylindrical vials $(5$ $\mathrm{cm}^{3}$ ) under Ar atmosphere, after several Ar purges. They were then mechanically ground in a Retsch MM2 horizontal vibratory mill operating at $25 \mathrm{~Hz}$ during time intervals from $30 \mathrm{sec}$ to several $\mathrm{h}$. The estimated milling intensity ${ }^{9}$ under these conditions was $450 \mathrm{~m} / \mathrm{s}^{2}$. Some samples were annealed for $1 \mathrm{~h}$ in dynamic vacuum $\left(p<3 \times 10^{-6} \mathrm{mbar}\right)$ at fixed temperatures ranging from $100-200{ }^{\circ} \mathrm{C}$.

After each milling run or annealing treatment the samples were characterized by XRD and ME spectroscopy. XRD patterns were obtained in reflection geometry using a Philips PW1710 diffractometer with $\mathrm{Cu} K \alpha$ radiation. ME experiments were carried out with a conventional spectrometer under transmission geometry, employing a ${ }^{57} \mathrm{CoRh}$ radioactive source. All these measurements were performed at room temperature.

Some selected samples were analyzed with a Perkin Elmer DSC7 calorimeter under dried Ar atmosphere and using $\mathrm{Cu}$ pans. Continuous heating experiments were done at different heating rates $10,20,40$, and $80 \mathrm{~K} / \mathrm{min}$. Isothermal calorimetric measurements at temperatures ranging from 90 to $160^{\circ} \mathrm{C}$ followed by conventional continuous heating scans were also performed.

\section{RESULTS}

\section{A. Milling}

XRD patterns obtained after various milling times $\left(t_{m}\right)$ are shown in Fig. 1. All the reflections expected for a bcc phase with $B 2$ ordering are clearly seen in the starting material but, as milling proceeds, the intensity of the superstructure reflections $(j+k+l$ odd $)$, decreases as a consequence of disorder. Additionally, all the peaks broaden, reflecting the particle and domain size reduction and the increase in stored microstrain. These diffractograms were analyzed using a full pattern fitting procedure DBWS-9411 (Ref. 10) that allowed the simultaneous refinement of the lattice parameter a, the site occupancy (from which the long range order parameter $S$ may be evaluated) and two variables determining the peak breadth. The peak shape was described by a modified Thompson-Cox-Hasting pseudo-Voigt profile ${ }^{10}$ where the Gaussian and Lorentzian breadths were, respectively, given by

$$
\Gamma_{G}=\left(U \tan ^{2} \theta+W\right)^{1 / 2} \text { and } \Gamma_{L}=X \tan \theta+Y / \cos \theta .
$$

$W$ and $X$ were fixed parameters related to the instrumental resolution while the fitted parameters $U$ and $Y$ were used to estimate respectively the unit volume deformation (microstrain $\varepsilon$ ) and the average size of coherent regions for diffraction $(L)$ :

$$
\begin{gathered}
L=0.89 \lambda / Y, \\
\varepsilon=0.5 \sqrt{U} .
\end{gathered}
$$

Figure 2(a) shows the evolution of these parameters with milling time. It is worth noticing that the observed reduction

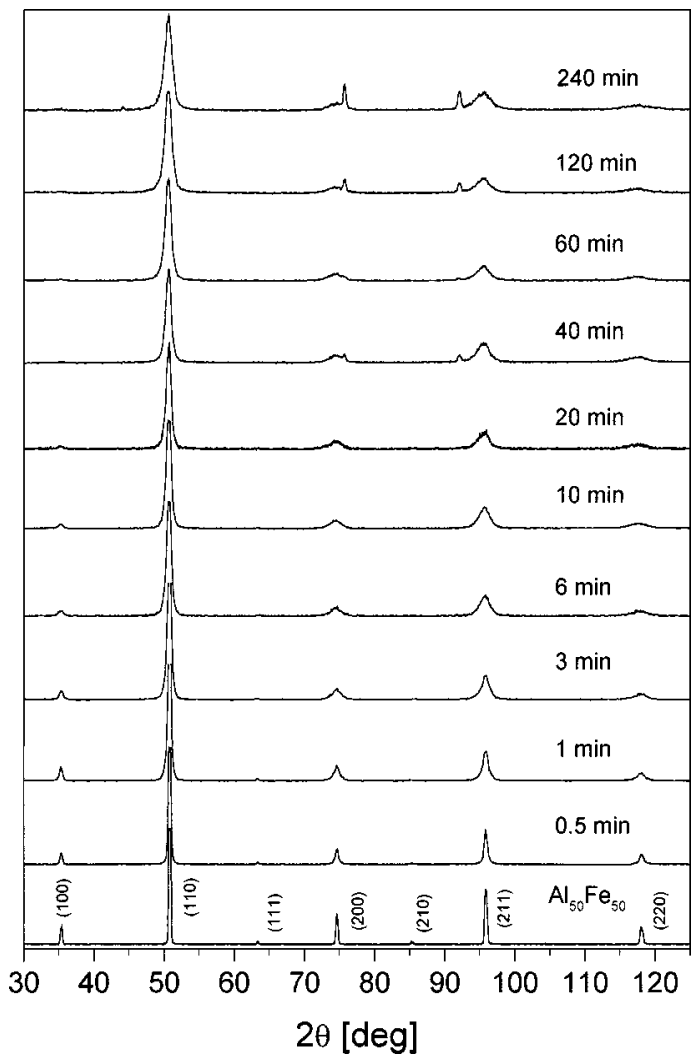

FIG. 1. X-ray diffractograms for different $t_{m}$. The extra peaks (not belonging to bcc) correspond to reflections of the sample holder (aluminum).

in grain size is not as severe as reported in previous work on similar systems,${ }^{2-4}$ probably due to the employed milling intensity. The lattice parameter and the strain increase rapidly in the first minutes of milling and then remains practically constant and the long range order parameter shows a smooth reduction toward its steady value.

ME spectra after different $t_{m}$ are displayed in Fig. 3(a). They all show a broad absorption peak at low velocities, characteristic of paramagnetic Al-Fe phases without any trace of ferromagnetic components. To analyze these unresolved doublets, several kind of fits were attempted using standard least squares routines and including either broadened Lorentzians, Voigt profile lines, ${ }^{5}$ or model-independent quadrupole splitting distributions (histograms). ${ }^{11}$ The best fits were obtained when the Mössbauer spectra were fitted with two components associated with the existence of two distinct regions with different degree of order. Both quadrupole interactions were described by a Gaussian distribution of Lorentzian doublets characterized by the isomeric shift $\delta$, the mean value of the quadrupole splitting $\Delta$, and its mean quadratic deviation $\sigma$. The first component, associated with the ordered zones, was constrained to have $\delta=0.288 \mathrm{~mm} / \mathrm{s}$ and $\Delta=0.0$ as in the starting unmilled sample. Its relative fraction $f$ was fitted. No constraints were imposed to the second component assigned to the less ordered zones.

The evolution of these parameters with milling time, are shown in Fig. 4(a). As the milling proceeds the fraction of the component asociated with disordered regions increases in detriment of the fraction associated with the ordered one. The value of $\sigma$ of the ordered component increases monoto- 


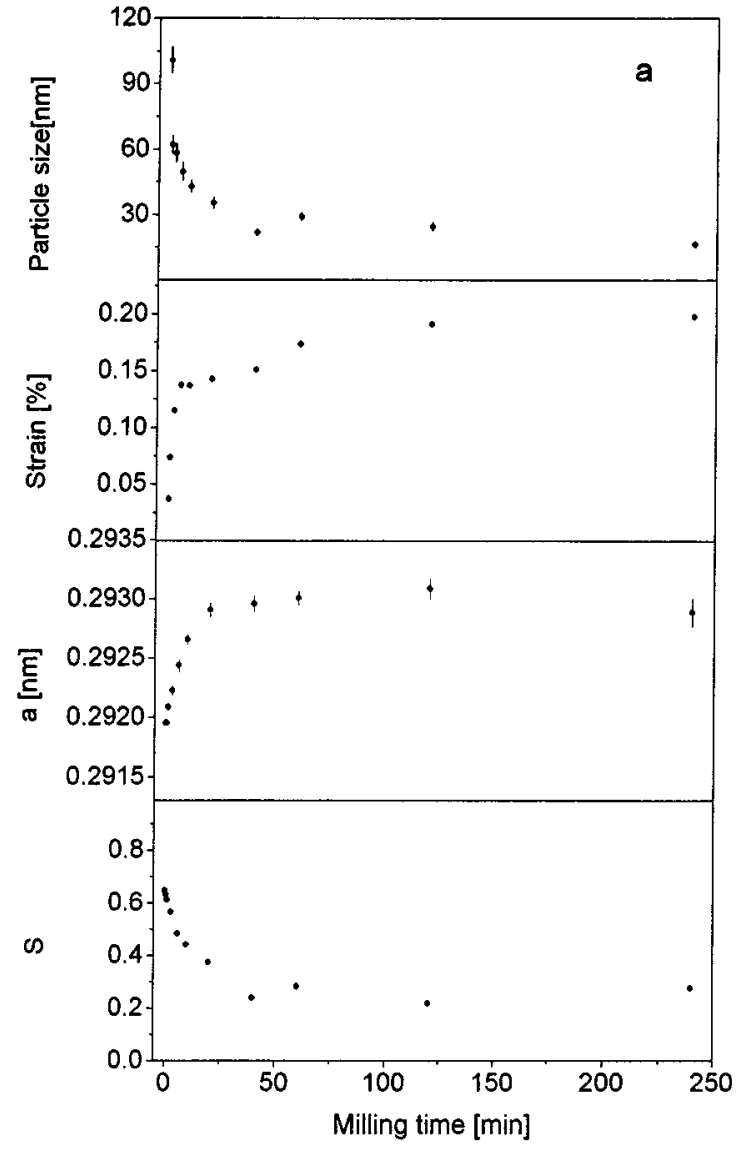

FIG. 2. (a) XRD fitted parameters vs $t_{m}$. (b) XRD fitted parameters vs $T_{\mathrm{ann}}$.

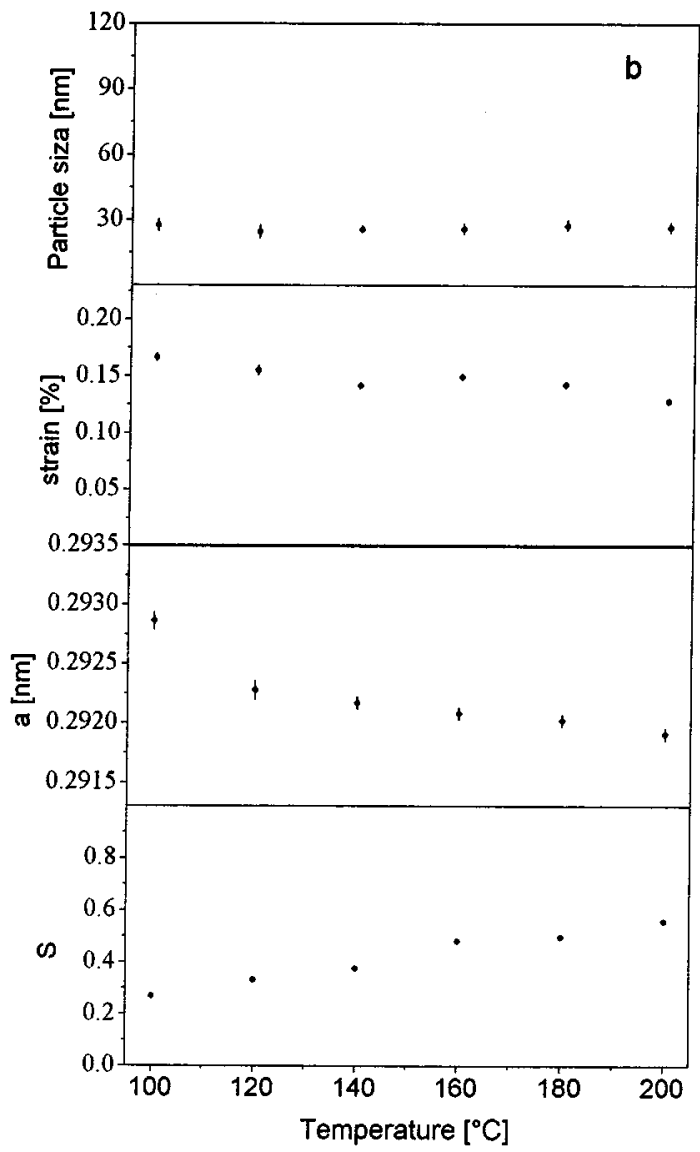

nously whereas those related to the disordered part remain almost constant. For milling times greater than $1 \mathrm{~h}$, both values are the same. The parameters associated with the disordered component $\left(\delta_{2}\right.$ and $\left.\Delta_{2}\right)$ evolve rapidly towards values close to those reported for a solid solution of AlFe. ${ }^{12}$

\section{B. Annealing}

Samples milled during 3, 6, 120, and 180 min have been analyzed by DSC in the continuous heating regime. The measured calorimetric signals at a heating rate of $40 \mathrm{~K} / \mathrm{min}$ are shown in Fig. 5 from room temperature up to $400{ }^{\circ} \mathrm{C}$. The main thermal accident occurs in the temperature range 110$250{ }^{\circ} \mathrm{C}$. It consists of an exothermic peak superimposed to a base line shift of the DSC signal. Both the area of the exothermic peak and the overall base line shift increase with $t_{m}$. The overall picture of the calorimetric behavior under heat treatment persists when a different value of the heating rate is used. The only change observed is an increase of the temperature $T_{p}$ of the peak maximum of the thermogram on increasing the heating rate, while the base line shift is not significantly affected. The apparent activation energy related to the observed exothermic peak evaluated by the Kissinger method and the base line shift as a function of $t_{m}$ are reported in Table I.

The time evolution of the calorimetric signal under isothermal regime was also measured at different selected temperatures $T_{a}$ in between those of the onset $T_{\text {on }}$ and of the peak maximum $T_{p}$ of the exothermic signal observed under continuous heating. In all these experiments the powders were heated from room temperature at a scan rate of 40 $\mathrm{K} / \mathrm{min}$ up to the isothermal temperature. The typical behavior observed under isothermal annealing is reported in Figs. 6 and 7. The exothermic heat flow shows a rapidly decreasing signal vs time, the amplitude of the signal being more important at increasing values of both $T_{a}$ and $t_{m}$.

To complement the kinetic analysis of the process, samples already annealed for $1 \mathrm{~h}$ at the selected temperatures $T_{a}$ were further submitted to continuous heating from room temperature to $400{ }^{\circ} \mathrm{C}$. Some of the DSC curves obtained are reported in Fig. 8, where the DSC curve of the as-milled sample is also included for comparison. From that figure it is apparent that after the isochronal annealing the heat evolved from the sample on further heat treating decreases on increasing $T_{a}$. However, the overall base line shift observed is of the same magnitude irrespective of the annealing temperature. That is, the complex calorimetric behavior depicted in Fig. 7 may be understood as the result of two processes, acting simultaneously in that temperature interval: (i) a reordering process and (ii) a recovery of the samples, as evidenced by XRD and MS experiments. Also, it may be concluded that after annealing at a given temperature $T_{a}^{\text {ref }}$ reordering is already achieved and only a remaining recovery effect is reflected in the DSC curve. One has $T_{a}^{\mathrm{ref}}=160^{\circ} \mathrm{C}$ in Fig. 8.

To get a feeling of the relative importance of the energies involved in these two processes, the respective values of 


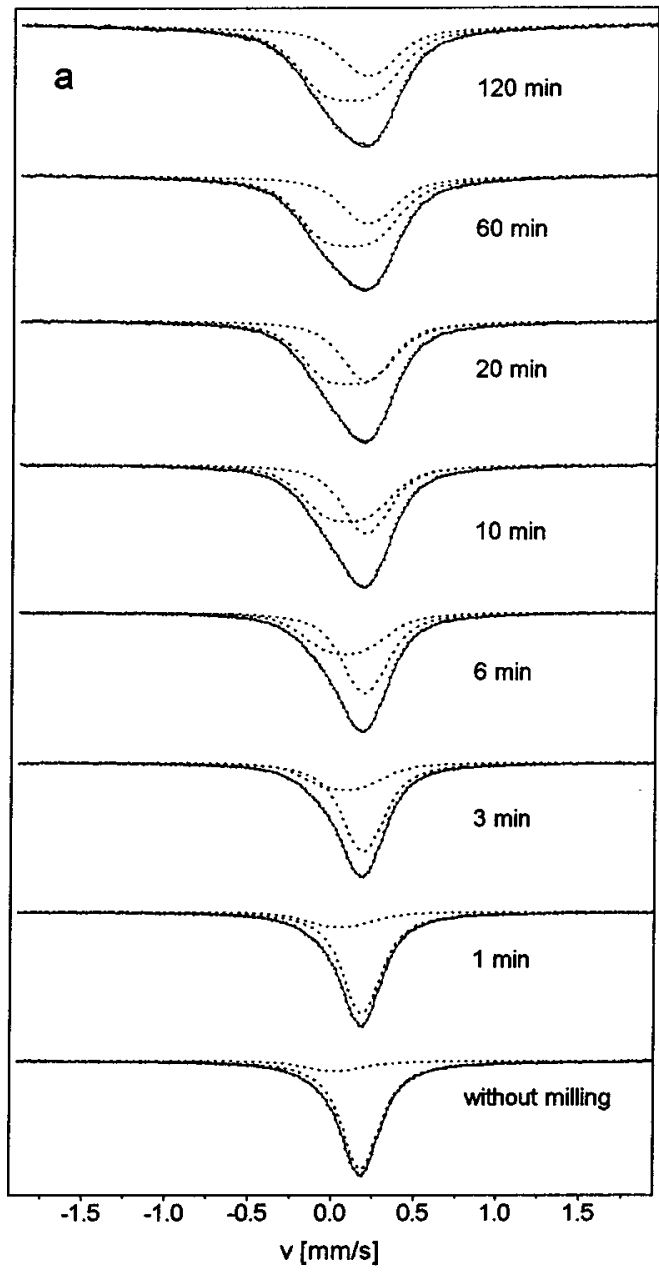

FIG. 3. (a) ME spectra for different $t_{m}$. (b) ME spectra for different $T_{\text {ann }}$.

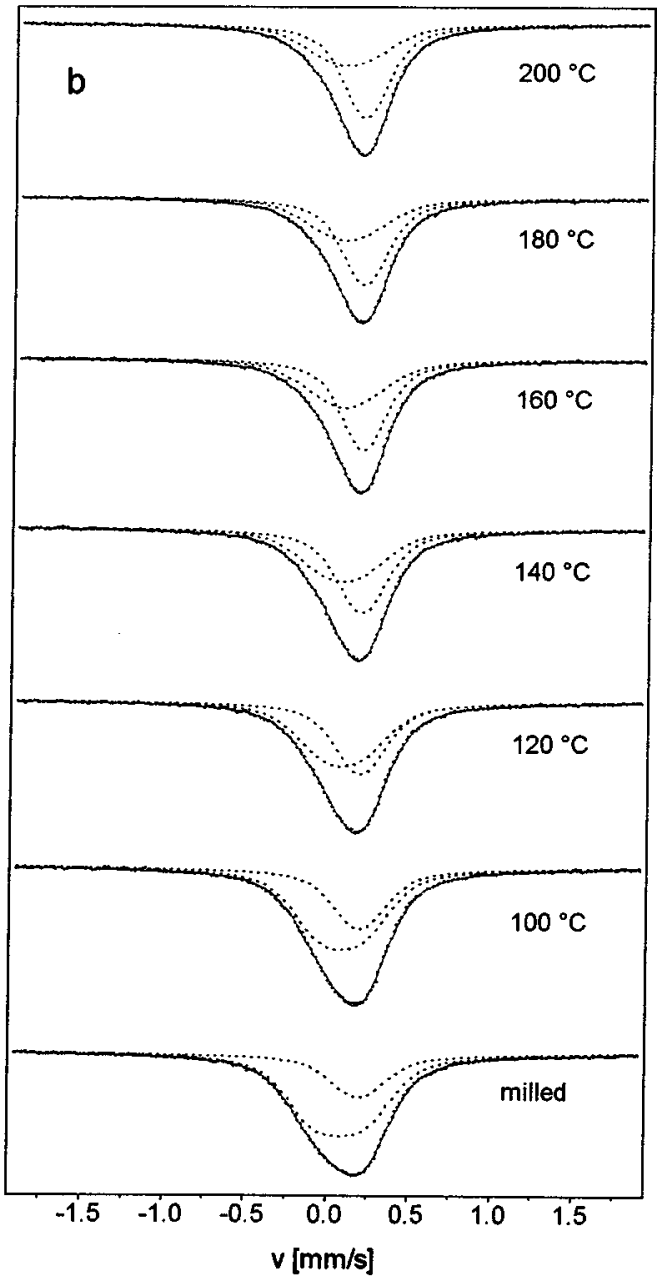

$\Delta H_{1}=\Delta H^{\text {iso }}\left(T_{a}\right), \quad \Delta H_{2}=\Delta H^{\text {heat }}\left(b T_{a}\right)-\Delta^{\text {heat }}\left(a T_{a}^{\text {ref }}\right), \quad$ and $\Delta H_{3}=\Delta H^{\text {heat }}\left(a T_{a}\right)-\Delta H^{\text {heat }}\left(a T_{a}^{\text {ref }}\right)$ were evaluated. The results are reported in Table II. Here $\Delta H^{\text {iso }}\left(T_{a}\right)$ stands for the total amount of heat evolved from the sample under isothermal annealing at Ta; $\Delta H^{\text {heat }}\left(b T_{a}\right)$ or $\Delta H^{\text {heat }}\left(a T_{a}\right)$ stand for the total amount of heat evolved from the sample under continuous heating before or after isothermal annealing for $1 \mathrm{~h}$ at $T_{a}$. As presented in Table II, a direct heating of the powder up to $250{ }^{\circ} \mathrm{C}$ results in a total enthalpy released of $36 \mathrm{~J} / \mathrm{g}$ with respect to that corresponding to a powder previously annealed $1 \mathrm{~h}$ at $160^{\circ} \mathrm{C}$. Such enthalpy change is attributed to the reordering process since it is also activated by isochronal annealing in the temperature range $110-160^{\circ} \mathrm{C}$. On the contrary, the recovery of the samples occurs in a much large temperature interval extending up to $250{ }^{\circ} \mathrm{C}$ as evidenced from the baseline shift detected by DSC. In view of this, we perform some ME and XRD measurements in annealed samples in order to follow the structural evolution during the above described thermal evolution to the equilibrium state.

The evolution of the XRD patterns with heat treatments can be observed in Fig. 9, where the progressive apparition of the superstructure peaks $(h+k+l=$ odd $)$ may be observed, denoting that some reordering takes place during the thermal treatments. On the other hand the full width at half maximum (FWHM) does not vary very much indicating that the mean cristallite size does not increase with the heat treatments.

The corresponding Mössbauer spectra are displayed in Fig. 3(b). They show a broad absorption peak corresponding to a distribution of unresolved quadrupole doublets that narrows with the thermal treatments. To fit these spectra we applied the same criteria as for the milled samples.

As can be seen in Fig. 4(b), the ordered component starts being the minor fraction of the Mössbauer spectra, but it becomes the majority as the annealing temperature increases. For the second component, the fitted values of $\delta_{2}$ and $\sigma_{2}$ remain almost constant and close to the values expected for a

TABLE I. Apparent activation energy $E_{A}$ and base line shift deduced from the DSC curves for different milling times $t_{m}$ together with the resulting long range order parameter. Note that the milling intensity for the sample milled $180 \mathrm{~min}$ was lower.

\begin{tabular}{rccc}
\hline$t_{m}(\min )$ & $S$ & $E_{A}(\mathrm{eV})$ & Base line shift $(\mathrm{J} / \mathrm{g} \mathrm{K})$ \\
\hline 3 & 0.69 & $1.22 \pm 0.15$ & $0.106 \pm 0.002$ \\
6 & 0.57 & $1.17 \pm 0.15$ & $0.133 \pm 0.002$ \\
180 & 0.40 & $1.19 \pm 0.15$ & $0.310 \pm 0.006$ \\
120 & 0.32 & $1.12 \pm 0.15$ & $0.320 \pm 0.006$ \\
\hline \hline
\end{tabular}




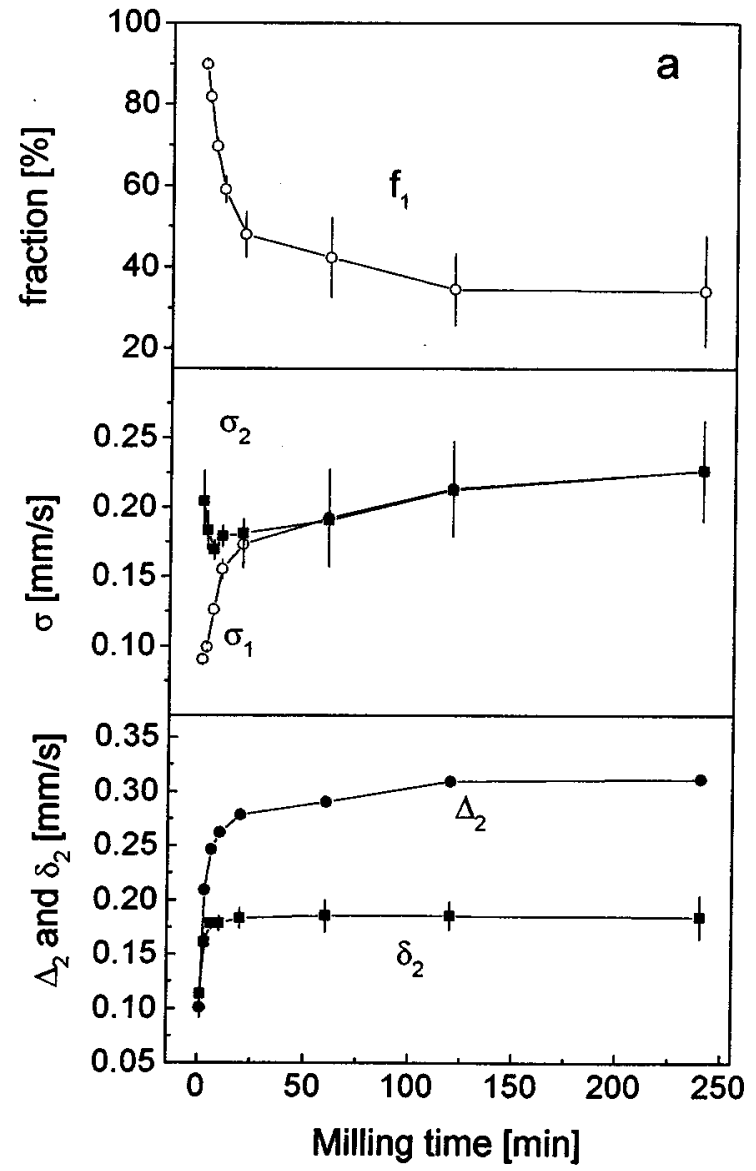

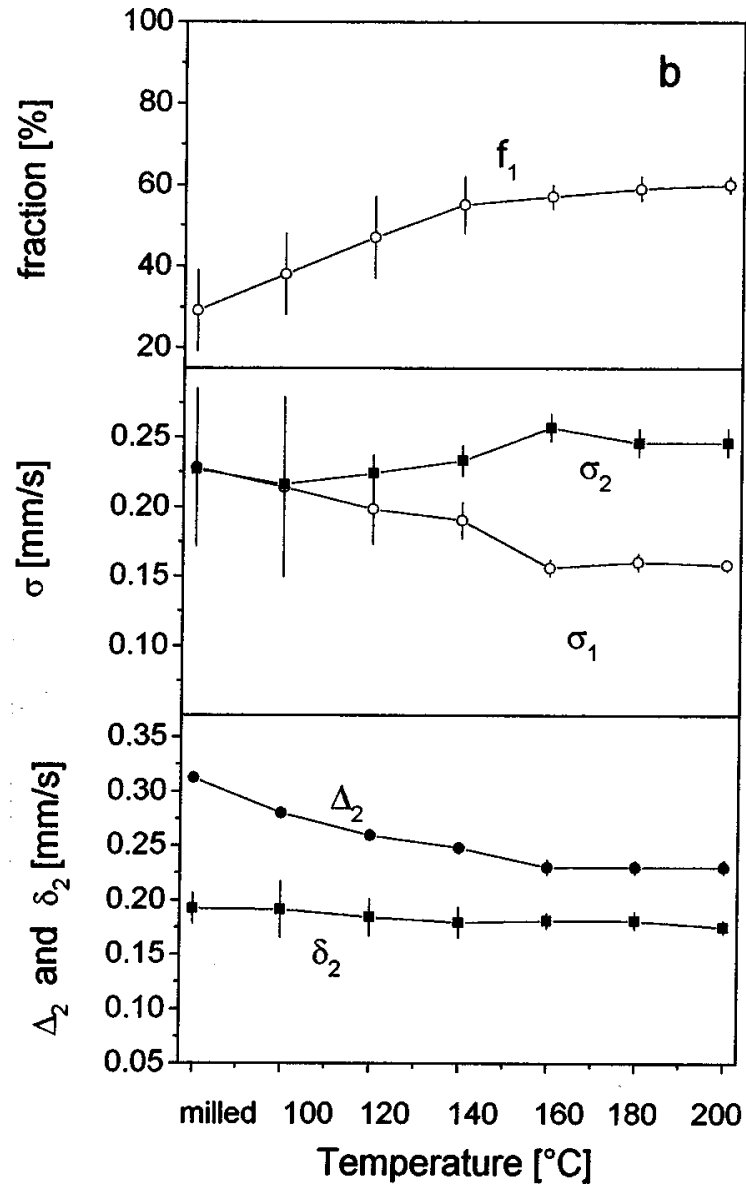

FIG. 4. (a) ME fitted parameters vs $t_{m}$. (b) ME fitted parameters vs $T_{\text {ann }}$.

bcc random solid solution AlFe. On the other hand, $\Delta_{2}$ decreases with the annealing temperature reflecting the fact that the electric field gradient at the Fe site decreases too, in good agreement with the reordering process.

\section{DISCUSSION}

As previously proposed, ${ }^{3}$ the LR disorder produced by fragmentation should increase linearly with $1 / L$. In the

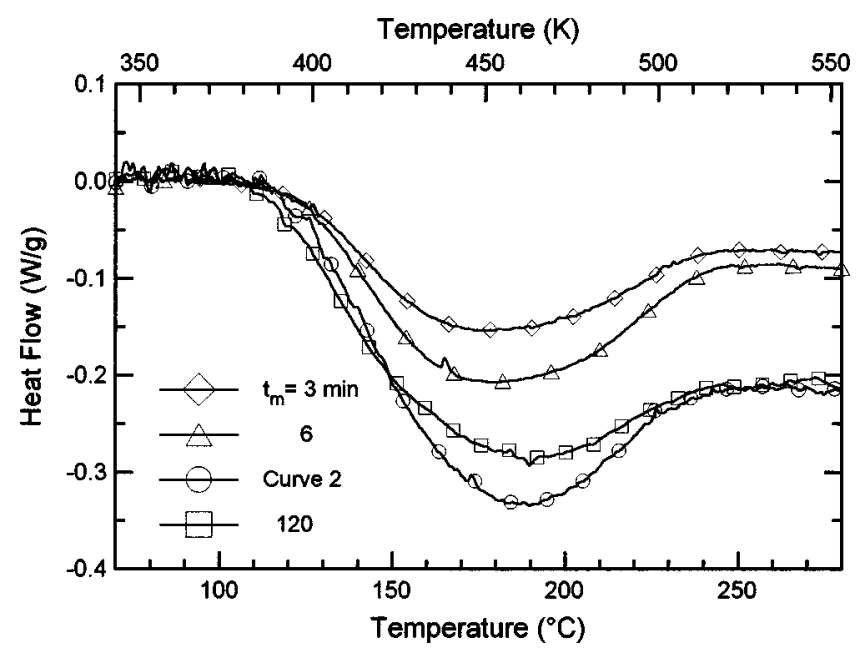

FIG. 5. DSC heating curves at $40 \mathrm{~K} / \mathrm{min}$ of the as-milled samples. present case $(1-S)$ scales with $1 / L$ only during the first few minutes of milling and afterwords a sudden drop in $S$ occurs with a simultaneous important increase in the accumulated microstrain $\varepsilon$, suggesting that lattice disorder is the main mechanism for the observed decrease in LR order. Then the reduction of the crystallite size cannot explain the observed variation of the LR order parameter with milling.

In the case of $B 2$ structure disorder is mainly due to vacancies and antisites. When vacancies predominate the lattice parameter decreases, while if the predominant defect is the antisites, the lattice parameter may increase or decrease depending on the misfit in the atomic volumes of the alloy

TABLE II. Values of the enthalpy released $\Delta H_{1}$ during isothermal annealing at $T_{a}$ and those released during a previous/post heattreatment, $\Delta H_{2} / \Delta H_{3}$, up to a recovery state defined by $1 \mathrm{~h}$ annealing at $T_{a}^{\mathrm{ref}}=160^{\circ} \mathrm{C}$, for samples milled for $t_{m}=180 \mathrm{~min}$.

\begin{tabular}{rrrr}
\hline \hline $\begin{array}{c}T_{a} \\
\left({ }^{\circ} \mathrm{C}\right)\end{array}$ & $\begin{array}{c}\Delta H_{1} \\
(\mathrm{~J} / \mathrm{g})\end{array}$ & $\begin{array}{c}\Delta H_{2} \\
(\mathrm{~J} / \mathrm{g})\end{array}$ & \multicolumn{1}{c}{$\begin{array}{c}\Delta H_{3} \\
(\mathrm{~J} / \mathrm{g})\end{array}$} \\
\hline & & & $36.2 \pm 3.0$ \\
90 & $2.5 \pm 0.2$ & & $34.8 \pm 3.0$ \\
100 & $8.3 \pm 1.0$ & & $28.2 \pm 3.0$ \\
110 & $10.8 \pm 1.4$ & $0.3 \pm 0.2$ & $25.3 \pm 2.6$ \\
120 & $16.5 \pm 1.8$ & $1.1 \pm 0.2$ & $18.9 \pm 2.0$ \\
130 & $21.6 \pm 2.0$ & $1.6 \pm 0.2$ & $13.1 \pm 1.5$ \\
140 & $24.9 \pm 2.5$ & $4.0 \pm 0.3$ & $7.0 \pm 1.0$ \\
150 & $25.7 \pm 2.6$ & $6.9 \pm 0.9$ & $3.9 \pm 0.7$ \\
\hline \hline
\end{tabular}




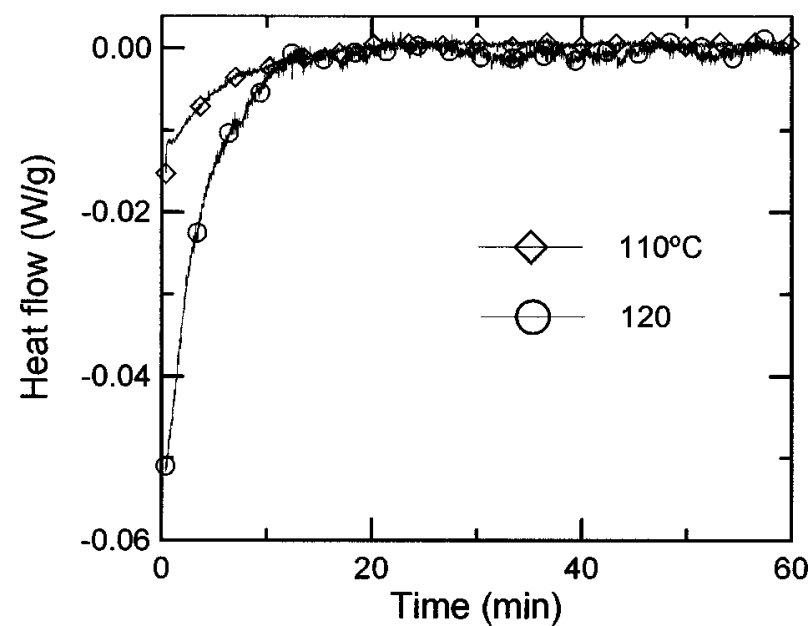

FIG. 6. DSC isothermal curves of samples milled for $t_{m}$ $=120 \mathrm{~min}$.

constituents. Then, in our case, the increase of the lattice parameter can be associated with the formation of antisites. This has been observed not only in AlFe and AlRu (Ref. 3) but also in $B 2-C o Z r .{ }^{13}$ The latter compound amorphizes under prolonged milling but, as shown by magnetization studies, disorder occurs before with antisistes as predominant defects. The formation of antisite defects by milling is consistent with the existence of this kind of defects in equilibrium at high temperature. In the case of $\mathrm{CoZr}$, probably the large missfit in atomic sizes leads to amorphization.

According to Edelin et al. ${ }^{14}$ we can associate the increment in the lattice parameter with the concentration of antisites as follows:

$$
a=a_{0}\left\{1+c_{\mathrm{AS}}^{a}\left(\Omega_{\mathrm{AS}}^{a}-1\right) / 3+c_{\mathrm{AS}}^{b}\left(\Omega_{\mathrm{AS}}^{b}-1\right) / 3\right\},
$$

where $\Omega_{\mathrm{AS}}^{a}\left(\Omega_{\mathrm{AS}}^{b}\right)$ is the relative volume of the vacancy with respect to the $a(b)$ element and $c_{\mathrm{AS}}^{a}\left(c_{\mathrm{AS}}^{b}\right)$ is the concentration of vacancies in the sublattice corresponding to $a(b)$ element

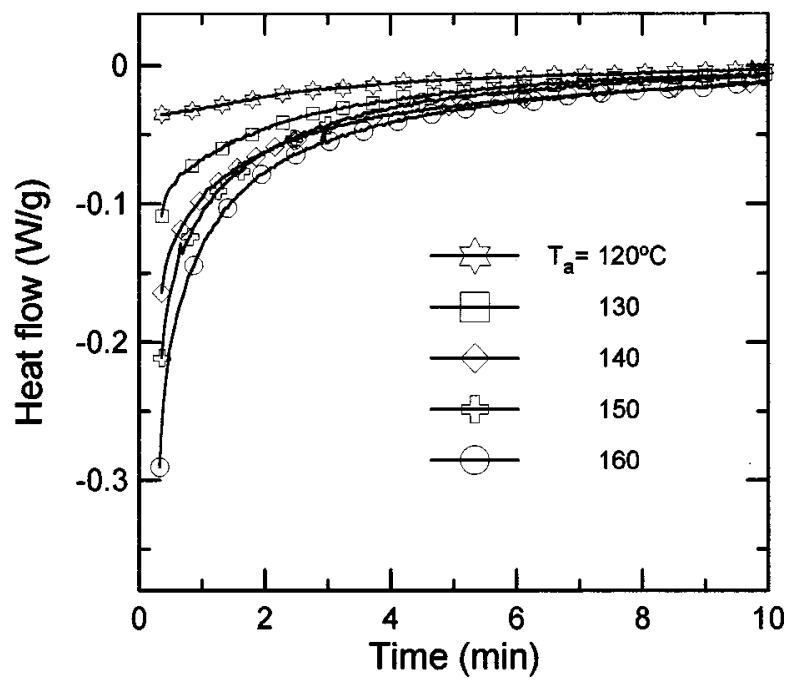

FIG. 7. DSC isothermal curves of samples milled for $t_{m}$ $=120 \mathrm{~min}$, during the first $10 \mathrm{~min}$ of annealing at several temperatures $t_{a}$.

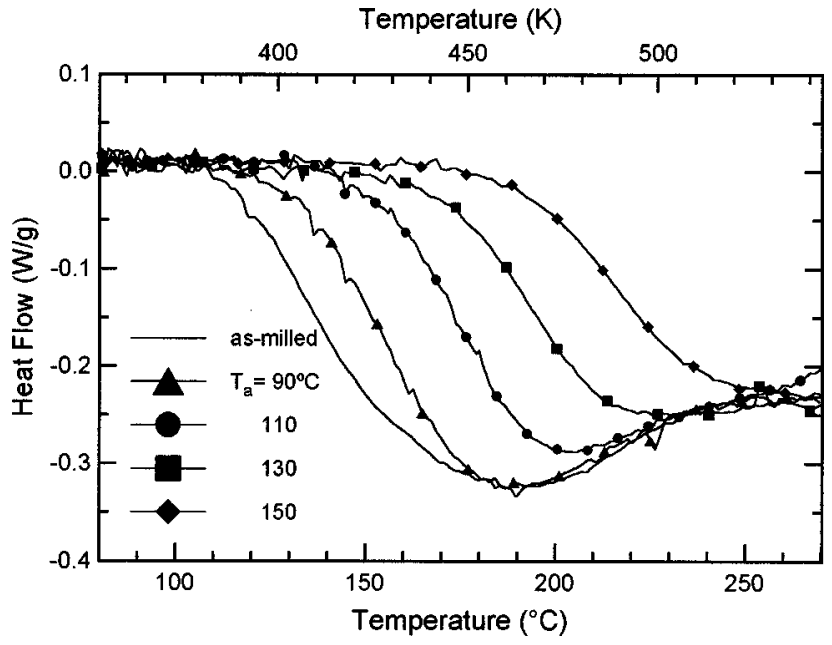

FIG. 8. DSC heating curves at $40 \mathrm{~K} / \mathrm{min}$ for as-milled samples and after annealing at several temperatures $t_{a}$.

Calculation made by $\mathrm{Fu}$ et al. $^{15}$ give that an aluminum antisite produces an expansion of $4 \%$ in the first coordination sphere, whereas an iron antisite produces a contraction of $1 \%$. Assuming an equal concentration of $\mathrm{Al}$ and $\mathrm{Fe}$ antisites $c_{\mathrm{AS}}$, the following relation betwen lattice parameter and antisite concentration is found:

$$
a=a_{0}\left\{1+0.07443^{*} c_{\mathrm{AS}}\right\},
$$

where $a_{0}=0.29196 \mathrm{~nm}$. From this formula and the observed dependence of the lattice parameter with $t_{m}$ we can calculate the rate of change of antisite concentration, in the first stages

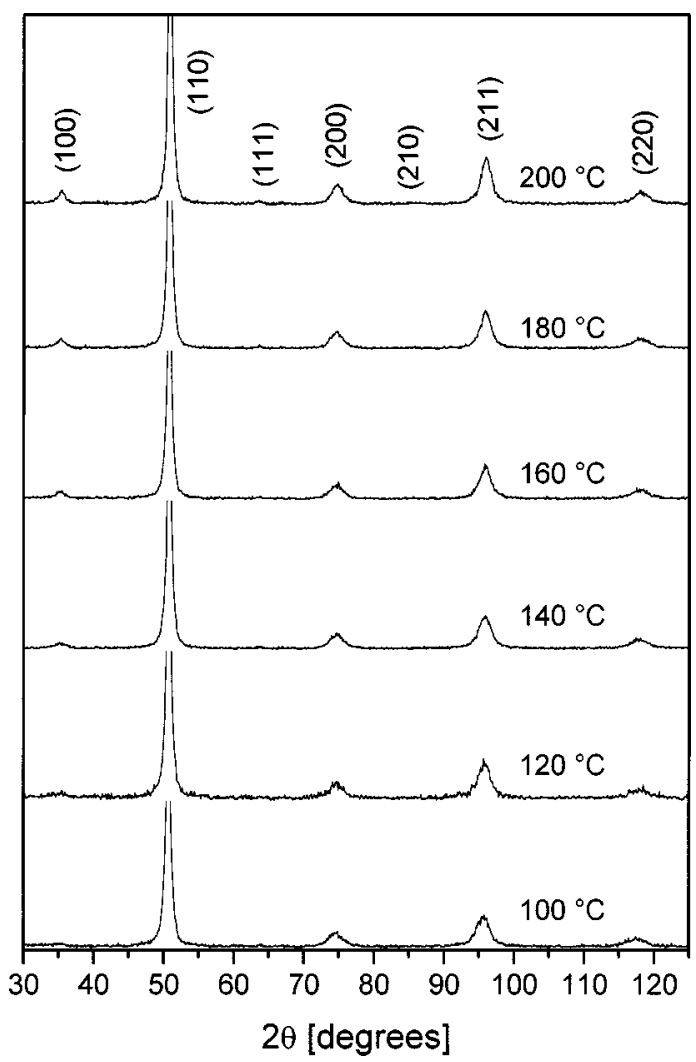

FIG. 9. X-ray diffractograms for different $T_{\text {ann }}$. 
of milling. We found for our milling conditions $\left(0.0033_{2} 1 / \mathrm{min}\right) 5.5 \times 10^{-5} \mathrm{~s}^{-1}$.

The disorder produced by milling in the $B 2$ structures is mainly due to the slip of $\{110\}$ planes in the $\langle 111\rangle$ direction, that produces the formation of antiphase boundaries (APB). ${ }^{16}$ The APB's are not true antisites but produces a similar effect on the lattice parameter because they put similar atoms together across the boundary.

These justify the model assumed to fit the Mössbauer spectra in term of two components, one associated with the ordered zones (constrained to have $\delta=0.288 \mathrm{~mm} / \mathrm{s}$ and $\Delta$ $=0.0 \mathrm{~mm} / \mathrm{s}$ as in the starting unmilled sample). Only its relative fraction $f$ and line with were fitted and no constraints were imposed to the second component assigned to the less ordered zones. The evolution of the ME can be interpreted as follows, the milling produces sliding planes that progressively disorder the alloy in such a way that the disorder is mainly concentrated in the sliding planes neighborhood (APB), whereas the interplanar regions remain practically unnaffected. The steady state reached may be the result of a saturation effect due to the fact that when these ordered regions rest inmerse in a disordered matrix, the next sliding plane practically produces no variation in the situation.

\section{CONCLUSIONS}

Room temperature milling of AlFe at an intensity of 450 $\mathrm{m} / \mathrm{s}^{2}$ leads to a steady state value of $S$ of about 0.32 . Al- though the particle size reduces during milling, this disorder is mainly due to lattice disorder, originated at the sliding planes produced by the milling process in this class of alloys. The SR order evolution is consistent with a nonstatistical order distribution throughout the sample but mainly distributed in two regions with different degree of order each. The highly disordered regions are those directly involved in the sliding planes whereas the most ordered ones are the unaffected zones trapped betwen sliding planes.

In the calorimetric studies a rapid evolution towards equilibrium is observed as two overlapped processes. Coupled continuous and isothermal treatment of the milled powders allowed us to separate the reordering and recovery processes. The kinetics of the reordering process is described in terms of already existing ordered regions which extend with an activation energy of $0.91 \mathrm{eV}$ and an enthalpy of 36.2 $\pm 3.0 \mathrm{~J} / \mathrm{g}$. In summary, the results are fully compatible with a mechanism of sliding planes that creates antiphase boundaries during the milling process.

\section{ACKNOWLEDGMENTS}

Support from CEE through Project No. CI1*-CT94-0029 (DG 12 HSMU), from CICYT (Spain) through Project No. MAT96-0692/0769, and from CONICET (Argentina) is acknowledged.
${ }^{1}$ G. P. Huffman and R. M. Fisher, J. Appl. Phys. 38, 735 (1967).

${ }^{2}$ P. Pochet, E. Tominez, L. Chaffron, and G. Martin, Phys. Rev. B 52, 4006 (1995).

${ }^{3}$ E. Hellstern, H. J. Fecht, Z. Fu, and W. L. Johnson, J. Appl. Phys. 65, 305 (1988).

${ }^{4}$ I. Börner and J. Eckert, Mater. Sci. Forum 225-227, 377 (1996).

${ }^{5}$ M. T. Clavaguera-Mora, J. Zhu, M. Meyer, L. Mendoza-Zélis, F. H. Sánchez, and N. Clavaguera, Mater. Sci. Forum 235-238, 541 (1997).

${ }^{6}$ M. Meyer, L. Mendoza-Zélis, and F. H. Sánchez, Hyperfine Interact. 2, 213 (1997).

${ }^{7}$ L. M. Di, H. Bakker, Y. Tamminga, and F. R. de Boer, Phys. Rev. B 44, 2444 (1991).

${ }^{8}$ P. Pochet, P. Bellon, L. Chafron, and G. Martin, Metastable Metal Based Phases and Microstructures, MRS Symposia Pro- ceedings No. 400 (Materials Research Society, Pittsburgh, 1995), p. 316.

${ }^{9}$ Y. Chen, M. Bibole, R LeHazif, and G. Martin, Phys. Rev. B 48, 14 (1993).

${ }^{10}$ R. A. Young, A. Sakthivel, T. S. Moss, and C. O. Paiva-Santos, Rietveld Analysis of X-ray and Neutron Powder Diffraction Patterns (User Guide to program DBwS-4911), Georgia Institute of Technology, 12, 1994, p. 12.

${ }^{11}$ R. A. Brandt, Nucl. Instrum. Methods Phys. Res. B 28, 398 (1987).

${ }^{12}$ J. H. Hsu and C. L. Chien, Hyperfine Interact. 69, 451 (1991).

${ }^{13}$ G. F. Zhou and H. Bakker, Mater. Trans., JIM 36, 329 (1995).

${ }^{14}$ G. Edelin, Acta Metall. 22, 455 (1978).

${ }^{15}$ C. L. Fu, Y. Y. Ye, M. H. Yoo, and K. M. Ho, Phys. Rev. B 48, $6712(1993)$

${ }^{16}$ M. J. Marcinkowsky and R. M. Fisher, J. Appl. Phys. 34, 2135 (1963). 